\title{
ARTIGOS
}

\section{LEISHMANIA MEXICANA: ASPECTOS TAXONÓMICOS Y RAREZA DE LA INFECCIÓN HUMANA EN TRÊS BRAÇOS, BAHIA, BRASIL ${ }^{1}$}

\author{
César Augusto Cuba Cuba, Air Colombo Barreto y \\ Philip Davis Marsden2
}

\begin{abstract}
Del estudio de 51 stocks de Leishmania aislados de pacientes humanos de leishmaniasis cutaneomucosa en Três Braços, Bahia, Brasil, los autores describen, en detalle, el análisis de los dos únicos stocks de L. mexicana, identificando uno de ellos como L. mexicana amazonensis El otro aislado permanece en posición taxonómica no definida pues considerándosele como un miembro de L. mexicana, encuéntranse dificultades para su identificación subespecifica. Evaluan también los parámetros biologicos e isoenzimáticos y discuten el papel de los anticuerpos monoclonales en la tipificación de éstos stocks.
\end{abstract}

Los autores remarcan la rareza de la transmisión de parásitos del complejo L. mexicana en esta región, aun cuando estudien epidemiológicamente por mais de 8 años la infección humana.

Palabras claves: Leishmania mexicana. L. mexicana amazonensis. Transmisión humana. Aspectos taxonómicos.

Durante los estudios epidemiológicos efectuados por nosotros en Três Braços, Bahia, reconocida área endémica de Leishmaniasis Cutaneomucosa, hemos aislado numerosas muestras de Leishmania de origen humano. Cuidadosa caracterización e identificación de esos parásitos ha sido realizada, empleándose critérios biológicos, bioquímicos e inmunológicos ${ }^{4}$. De las 51 muestras aisladas de humanos con lesiones cutáneas, 49 (96\%) fueron tipificadas como siendo Leishmania braziliensis braziliensis, razón por la cual consideramos ésta área como de casi exclusiva transmisión humana por éste parásito.

Motivo de la presente comunicación constituye el estudio de los otros dos aislados, los únicos no tipificados como L. b. braziliensis describe los casos, clínicos, la evaluación biológica de los organismos, los resultados de los estudios isoenzimáticos e inmunológicos y discute las dificultades encontradas en la aplicación de algunos parámetros de identificación de una de las muestras en caracterización.

1. Trabajo parcialmente financiado por el CNPq PIDE V 403682 y WHO/TDR-RE. Grant Special Programme for Research and Training in Tropical Diseases.

2. Faculdade de Ciências da Saúde e Núcleo de Medicina Tropical - Universidade de Brasília - Brasilia - DF Brasil.

Recebido para publicação em 18/7/84.

\section{MATERIAL Y MÉTODOS}

\section{Observaciones Clínicas}

Paciente 1. I. S., niña de 14 años de edad, que tenia una lesión cutánea ulcerada de $3 \mathrm{~cm}$ de diámetro, localizada en el tercio medio de la pierna derecha, con un mes de duración. En términos clínicos la lesión no pudo ser diferenciada de los casos cutáneos producidos por L. braziliensis braziliensis.

La intradermorreación de Montenegro fue positiva con $12 \mathrm{~mm}$ de diametro. El test de inmunofluorescencia indirecta (IFI) fue negativo y se mantuvo asi sistemáticamente durante todo el seguimiento (más de 6 años después del tratamiento). La paciente fue tratada con Nifurtimox (Lampit), por Marsden ${ }^{11}$, observándose respuesta excelente y completa cicatrización de la herida.

Paciente 2. M. J. S. Un stock de Leishmania fue aislado en Julio de 1978 de este niño de 11 años de edad procedente de una aislada región rural en la Hacienda Taboquinha, $15 \mathrm{~km}$ al sureste de Três Braços. El paciente tenia 6 lesiones cutáneas simultaneamente, en los brazos y tronco, todas ellas con aproximadamente 4 meses de duración. Los tests diagnósticos de rutina mostraron lo siguiente: prueba cutánea de Montenegro positiva, frotices de las 
Cuba CAC, Barreto AC, Marsden PD. Leishmania mexicana: aspectos taxonómicos y rareza de la infección humana en Três Braços, Bahia, Brasil. Revista da Sociedade Brasileira de Medicina Tropical 17: 115-122, Jul-Set, 1984

lesiones negativa para amastigotas; histopatologia con la dermis fuertemente infiltrada com células plasmáticas, linfócitos y macrófagos, no fue visto granuloma; polimorfonucleares estaban presentes. La serología presentó un título de IFI de 80 . La inoculación intradérmica de hamsters (Mesocricetus auratus) reveló formas amastigotas del parásito.

\section{Observaciones de Laboratorio}

Ambos stocks de Leishmania fueron aislados por la inoculación en la nariz y patas posteriores de hamsters, de un homogeinizado de tejidos de las biopsias de los pacientes, siendo identificados con el código LTB-0016 (Paciente 1) y LTB-0055 (Paciente 2), respectivamente. Despues de criopreservados $\left(-196^{\circ} \mathrm{C}\right)$ fueron depositados en el banco de cepas del Laboratorio de Protozoología de la Universidade de Brasilia, Brasil.

Los siguientes parámetros para la identificación fueron estudiados:

a) Comportamiento del parasitismo en los hamsters incluyendo las caracteristicas de desarrollo de la lesión, evolución e histología.

b) Morfologia de amastigotas. Frotices de las lesiones experimentales fueron fijados en fluido de Bouin y coloreados por Giernsa. Los amastigotas fueron medidos (dimensiones del largo por ancho) a partir de las microfotografias tomadas de las láminas coloreadas, de acuerdo a lo reportado por Shaw \& Lainson ${ }^{16}$. Preparaciones coloreadas de las cepas padrón de L. mexicana amazonensis (PH8) y L. braziliensis guyanensis (M 4147) obtenidas de R. Lainson, fueron utilizadas para la comparación morfológica y biométrica.

c) Comportamiento en medio $3 \mathrm{~N}(\mathrm{~N} \mathrm{~N} \mathrm{~N})$ y en otros medios de cultivo. Utilizamos los medios líquidos de LIT (liver infusion tryptose) y de Schneider (Drosophila revised medium) y el bifásico de Difco (USAMRU ${ }^{19}$ ), con $15 \%$ de sangre desfibrinada de conejo.

d) Padrón de desarrollo en el tubo digestivo de Lutzomyia longipalpis.

Los insectos fueron obtenidos de la colonia de la Wellcome Parasitology Unit, Belém, Pará y de nuestra colonia de la Universidade de Brasilia. Cincuenta (50) hembras de Lu. Longipalpis fueron puestas a alimentar, a través de la piel de pollitos de 3 días de nacidos, en una mezcla de sangre de conejo inactivada y cultivo del stock LTB-0055 20 . Paralelamente, otro lote de insectos era puesto a succionar directamente sobre las lesiones no ulceradas de hamsters infectados con LTB-0055, de acuerdo a la metodología de Lainson ${ }^{7}$. Los flebótomos fueron disectados entre los 5 y 7 dias después de alimentados y el tubo digestivo examinado con microscopio de contraste de fases. Los critérios de evolución descritos por Lainson \& Shaw ${ }^{8}$ en el insecto fueron tomados en consideración.

e) Mobilidad electroforética de las isoenzimas.

Para la preparación de los lisados de promastigotas, cultivamos los parásitos en frascos de $250 \mathrm{ml}$ de capacidad, conteniendo $50 \mathrm{ml}$ de agar sangre Difco. Los promastigotas crecieron en $5-6 \mathrm{ml}$ del sobrenadante (solución salina físiológica). Un frotis coloreado por Giemsa fue preparado de los organismos cosechados "in vitro" para la confirmación de la morfologia típica y ausencia de contaminación.

Los estudios isoenzimáticos fueron ejecutados por el examen de la mobilidad electroforética, en gel fino de almidón, de 10 enzimas: aspartato aminotransferasa E.C. 2.6.1.1. (ASAT); alanino aminotransferasa E.C. 2.6.1.2 (ALAT), fosfoglucomutasa E.C. 2.7.5.1 (PGM); glucosa fosfato isomerasa E.C. 5.3.1.9 (GPI); malato dehidrogenasa E.C. 1.1.1. $37(\mathrm{MDH})$; enzima málica E.C. 1.1.1.40 (ME); glucosa - 6 - fosfato dehidrogenasa E.C. 1.1.1.49 (G6PD); amino peptidasa (cytosol) E.C. 3.4.11.1. (PEP); manosa fosfato isomerasa E.C. 5.3.1.8. (MPI) y fosfatasa acida E.C. 3.1.3.2. (ACP).

La metodologia fue llevada a cabo siguiendo esencialmente la descrita por Miles et al ${ }^{13}$, excepto para las siguientes ligeras modificaciones del tiempo y voltaje, en la electroforesis: ASAT y ALAT, 150' y 300 voltios; GPI, ACP, PGM, MDH, MPI, PEP, G6PD, ME, 150' y 200 voltios. Los perfiles isoenzimáticos de las leishmanias fueron comparados en la misma placa electroforética, con los siguientes extractos correspondientes a las cepas de referencia del laboratorio de la Wellcome Parasitology Unit (Belém), del Dr. Lainson Leishmania mexicana amazonensis ( $\mathrm{PH} 8)$, Leishmania braziliensis braziliensis (M4035), L. braziliensis guyanensis (M4147) y dos cepas de L. braziliensis braziliensis de Três Braços (LTB-0042 y LTB-0096) tipificadas como tales por isoenzimas y anticuerpos monoclonales. 
Cuba CAC, Barreto AC, Marsden PD. Leishmania mexicana: aspectos taxonómicos y rareza de la infección humana en Três Braços, Bahia, Brasil. Revista da Sociedade Brasileira de Medicina Tropical 17: 115-122, Jul-Set, 1984

\section{RESULTADOS}

\section{a) Comportamiento en Hamsters}

El stock LTB-0016 fue fácilmente aislado de las lesiones nodulares de los hamsters. Grandes histiocitomas fueron producidos constantemente por el parasitismo de éste stock. Sistematicamente el tiempo de incubación fué de 40 dias, en media, calculado de los diversos pasajes (hamster para hamster) del material de las respectivas lesiones experimentales.

La metástasis fue un fenómeno constante, presentándose en localizaciones diversas tales como base de la cola, bolsas escrotales, patas anteriores y orejas. La visceralización, en bazo e higado, también fue establecida. El parasitismo fué intenso y los amastigotas fueron típicamente de $L$. mexicana. En los cortes histológicos de los histiocitomas numerosos macrófagos llenos de parásitos fueron siempre observados (Figura 1).

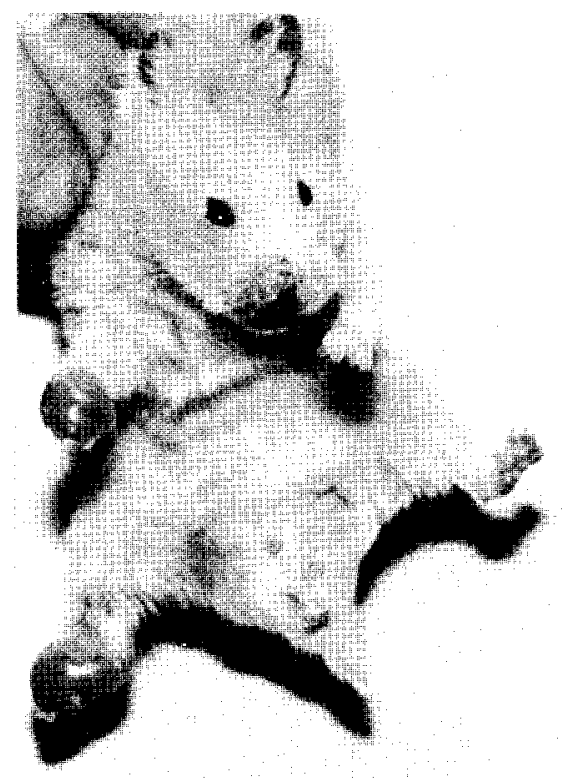

Fig. 1 - Fotografia de las lesiones desarrolladas por la muestra LTB-0016, Leishmania mexicana amazonensis, 45 dias después de -inoculada. Nótese la formación de grande lesión parasitaria metastásica en la pata anterior.

El cuadro parasitario descrito es caracteristico de las infecciones por parásitos del complejo L. mexicana y contrastó con la evolución del parasitismo observado con el stock LTB-0055, cuyo aislamiento primário fue obtenido de um hamster que no mostró lesiones en los locales de inoculación (nariz y patas posteriores) durante 216 dias después de inoculado. Sin embargo, cuando el animal fue necropsiado, los frotices coloreados del bazo mostraron abundantes amastigotas que, cuando inoculados en un segundo hamster desarrollaron nódulos subcutáneos, 2 meses después de la infección, no ulcerativos y eritematosos. Cinco pasajes de hamster para hamster de los amastigotas de éste stock no alteraron la evolución del parasitismo en dichos animales.

La evolución de la infección no mudó macroscópicamente. No se observó metástasis cutanea y la visceralización fué marcante con abundantes parásitos en el bazo y en menor número en el hígado.

En los frotices y cortes histológicos de las lesiones del hamster (LTB-0055) fueron observados número moderado de parásitos. La biometria de las formas amastigótas estableció los siguientes resultados: longitud $4.3 \times 2.9 \mu \mathrm{m}(\overline{\mathrm{X}} 3.2 \mu \mathrm{m})$ y anchura, $2.5 \times 2.2 \mu \mathrm{m}(\overline{\mathrm{X}} 2.3 \mu \mathrm{m})$. Biometricamente son compatibles con los de L. mexicana.

b) Comportamiento "in vitro" en medio $3 \mathrm{~N} \mathrm{y}$ otros medios.

Ambas leishmanias (LTB-0016 y LTB-0055) mostraron rápido y explosivo crescimiento después del aislamiento primário cuando sembradas en agar sangre simples (NNN, $15 \%$ de sangre de conejo desfibrinada), agar sangre Difco, USAMRU y LIT. En medio liquido de Schneider (suplementado con $20 \%$ de suero bovino fetal), $17-30 \times 10^{8}$ promastigotas por $\mathrm{ml}$ fueron facilmente obtenidos, con 5 días de crescimiento.

c) Comportamiento en el tubo digestivo de $L u$. longipalpis.

Esfuerzos fueron hechos para infectar $50 \mathrm{Lu}$. longipalpis empleando los promastigotas de LTB0055. Los 23 ejemplares bien alimentados que fuimos capaces de examinar, posteriormente, fallaron para infectarse y permitir el desarrollo de éste stock. Esto aconteció tanto cuando usamos el procedimiento de infección por alimentación de formas de cultivo como cuando alimentadas directamente, en las nódulos parasitarios no ulcerados de las infecciones experimentales. (15 insectos examinados de éste lote). 
Con LTB-0016, un tipico padrón de evolución suprapilário fue consistentemente obtenido en $L u$. longipalpis. Infecciones masivas por los parásitos se distribuyeron por estómago, cardias, esófago de los insectos, pero siempre respetando el triángulo posterior.

d) Análisis de las isoenzimas.

Los siguientes resultados fueron obtenidos del análisis de los perfiles isoenzimáticos conseguidos por la electroforésis de 9 de las 10 enzimas empleadas (ME no presentó resultados conclusivos):

1 - Por comparación, en la misma placa electroforética, los perfiles enzimáticos obtenidos con el stock LTB-0055 fueron distintos de $L$. braziliensis braziliensis (LTB-0042, LTB-0096 y M4035), de $L$. braziliensis guyanensis (M 4147) e indistinguible de L. mexicana amazonensis (PH8), con las enzimas ASAT, ALAT, MDH, MPI, PEP y GPI. La Figura 2 presenta los diagramas y las respectivas fotografías tomadas de las placas electroforéticas.
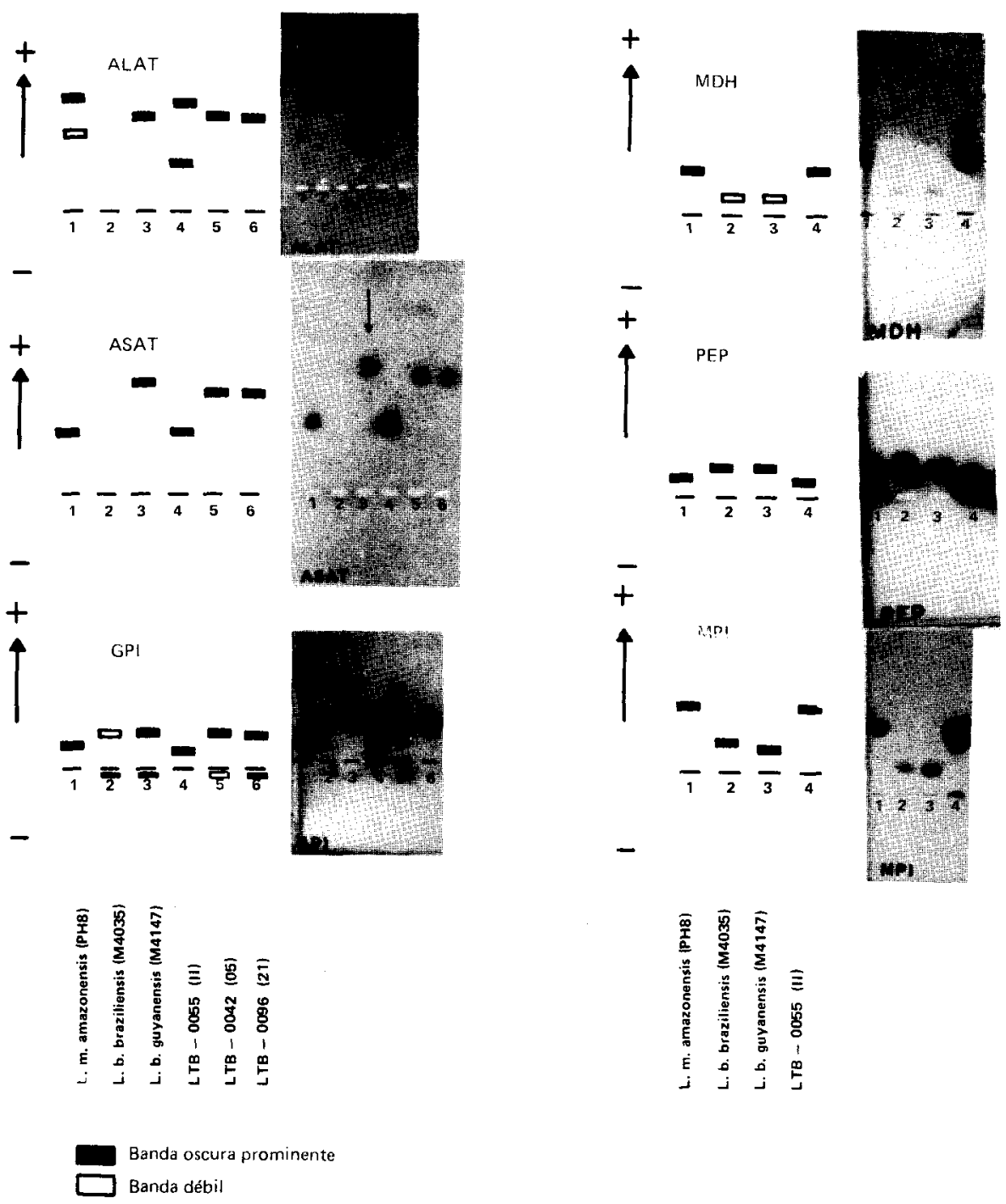

Fig. 2 - Representaciones diagramáticas de la posición de las bandas obtenidas con las enzimas ALAT ASAT, GPI, MDH, PEP y MPI y fotografias de los respectivos padrones electroforeticos. La flecha señala, en ALAT y ASAT, la posición de $L$. b. guyanensis como referencia para LTB-0055, pues el padrón de $L$. braziliensis (M4035) no pudo ser revelado. 
2 - Siete enzimas (ASAT, ALAT, MDH, G6PD, MPI, ACP y GPI) identificaron el stock LTB-0016, como isoenzimáticamente indistinguible de $L$. mexicana amazonensis (PH8) (resultados no presentados).

3 - Una banda prominente de desplazamiento electroforético rápido fue el perfil enzimático obtenido con LTB-0055 y G6PD, cuando comparado con las dos bandas más lentas presentadas por L. mexicana amazonensis (Figura 3). LTB-0055 es una $L$. mexicana, próxima a $L$. mexicana amazonensis.
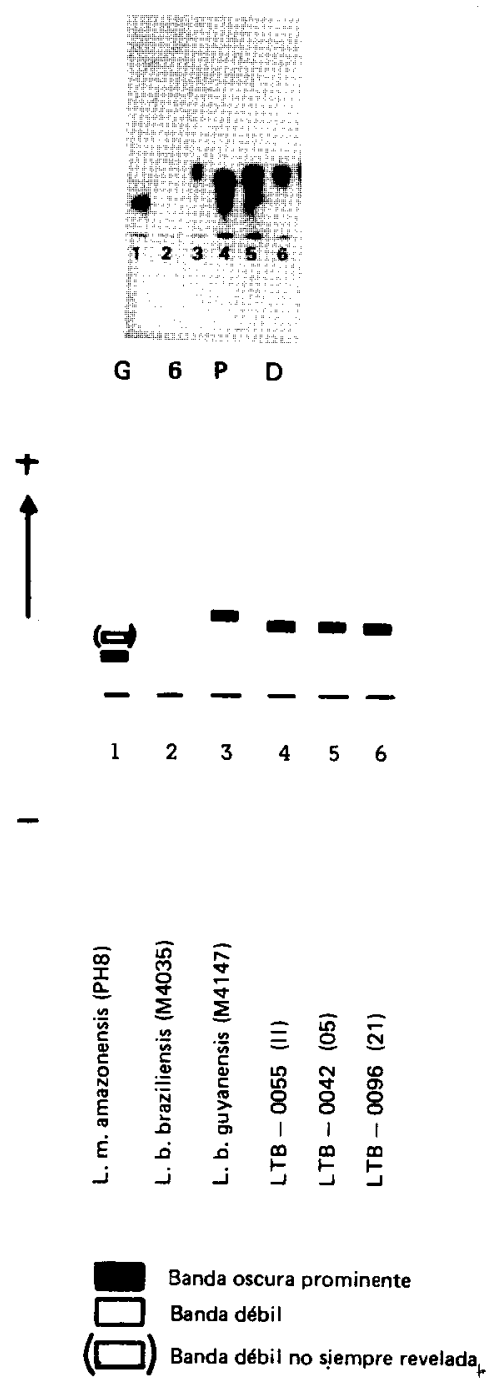

Fig. 3 - Representación diagramática y fotografia de la posición de la banda electroforética obtenida con la enzima G6PD y el stock LTB-0055 cuando comparada con las cepas de referencia.

\section{DISCUSION}

En áreas endémicas de leishmaniasis mucocutánea es generalmente considerado, sobre evidencias únicamente clinicas, que el parásito agente etiológico de la enfermedad sea Leishmania braziliensis. Lesiones cutáneas muy recientes se presentan en localidades con transmisión activa y constante, como es el caso de ésta extensa área endemica en el Estado de Bahia. En ésta situación epidemiológica es necesaria la correcta identificación de los parásitos, pues leishmaniasis cutánea puede ser también causada por parásitos del complejo $L$. mexicana. Certas lesiones clinicamente son indiferenciables de las causadas por L. braziliensis. Las implicaciones prognósticas y terapéuticas son evidentes para el clínico que estudia éstos pacientes.

Afirmase que el parasitismo humano por $L$. mexicana amazonensis es sumamente raro ${ }^{9}$ sin embargo otras subespecies de $L$. mexicana estarian causando infecciones humanas en el Valle del Rio Doce, Caratinga, Minas Gerais, Brasil ${ }^{14}$.

La área endémica de Bahia todavia presenta ecótopos en que algunas asociaciones biológicas aparentemente se mantienen: $L$. mexicana amazonensis, $L$. mexicana sp y Proechimys inheringi denigratus $^{1}$ y también la presencia, en densidades variables, de $L u$. flaviscutellata, conocido único vector de $L$. mexicana amazonesis ${ }^{10}$. Todos esos elementos de la cadena epidemiológica se encuentran en pequeños focos de florestas poco perturbadas, con árboles altos y en "capoeiras". Probablemente, la mudanza de los hábitos de los flebótomos contribuya para el surgimiento de casos esporádicos de parasitismo humano por L. mexicana, como es el caso de los pacientes estudiados en este trabajo. La existencia de especies de flebotomineos altamente antropofilicos, en Três Braços, abre la posibilidad de que tales insectos sean transmisores de leishmanias no identificadas (Barreto el al, en preparación).

Nuestra paciente no 1 (LTB-0016) parasitada por $L$. mexicana amazonensis acostumbraba a trabajar en un microfoco de Lu. flaviscutellata, próximo a la pequeña chacra en que sus padres laboraban. Lo descrito es sugestivo de la probable transmisión del parásito en éste ambiente modificado por el hombre.

Por otro lado, vários son los métodos actualmente disponibles que permiten la caracterización e identificación de los parásitos del género Leishma- 
Cuba CAC, Barreto AC, Marsden PD. Leishmania mexicana: aspectos taxonómicos y rareza de la infección humana en Três Braços, Bahia, Brasil. Revista da Sociedade Brasileira de Medicina Tropical 17: 115-122, Jul-Set, 1984

nia ${ }^{17}$. Entretanto, algunos procedimientos continuán todavia siendo técnicas sofisticadas de laboratorios de investigación bien equipados (Hibridización del ADN, enzimas de restricción); otras técnicas, más accesibles, son de práctico empleo en laboratorios de los trópicos (isoenzimas, anticuerpos monoclonales usados como reactivos serológicos en IFAT y inmunoperoxidasa) y tambièn, la caracterización biológica "in vivo" (evolución en flebótomos y hamsters) e "in vitro" (cultivos) ${ }^{6}$.

Cuando se pretende evaluar las características fenotipicas y genotípicas de los stocks de Leishmania obtenidos de pacientes, vectores y reservatorios, algunas dificultades ocurren en su interpretación. Aparentemente esos problemas taxonómicos estarían relacionados con la falla de algunos "marcadores" inmunobiológicos (anticuerpos monoclonales) e isoenzimáticos, para tipificar ciertos parásitos. Esto podria ser comprensible, pues consideráse que los parásitos pertenecientes al género Leishmania se encuentran en una fase de evolución y constante especiación.

Muy recientemente nuestro grupo ${ }^{1}$ ha encontrado dificultades para la identificación subespecifica de stocks de Leishmania aislados de Proechimys inheringi denigratus, roedores capturados en Três Braços. Los parásitos fueron distintos de L. mexicana mexicana y de $L$. mexicana amazonensis cuando provados por la IFAT, utilizando una batería de anticuerpos monoclonales. Isoenzimáticamente los mismos tres stocks, no pudieron ser distinguidos unos de otros con 9 enzimas y parecen ser próximos a $L$. mexicana amazonensis de la cual se separan con las enzimas GPI, PEP y ALAT.

Nuestro stock humano LTB-0055 presentó un comportamiento peculiar aparentemente mixto, participando de las caracteristicas de ambos complejos (L. mexicana y L. braziliensis). Falló para reunir los critérios que definen $L$. mexicana amazonensis. Asi, biológicamente presentó un desarrollo relativamente lento en los hamsters inoculados y no detectamos histiocitomas ni metástasis de los parásitos. Comprendemos las limitaciones de las observaciones "in vivo" que incluyen potencialmente problemas de variación de temperatura, en Brasília, afectando el bioterio, y a la probable influencia genética que los hamsters, no isogenicos, podrian ofrecer (Bradley) ${ }^{2}$; por ejemplo los animales reconociendo sólo algunos antigenos (Perez) ${ }^{15}, \mathrm{y}$, a la variación genética que los propios parásitos (LTB-0055) podrían presentar.
El fracaso en desarrollar LTB-0055 en el tubo digestivo de $L u$. longipalpis, no puede ser atribuido a un prolongado tiempo de cultivo "in vitro". El aislado tenía pocos subcultivos y además, falló para infectar los insectos como amastigotas en la lesiones de los hamsters. No pudimos establecer entonces el padrón Suprapilario ${ }^{8}$ característico de los parásitos del complejo L. mexicana. Usualmente, las infecciones con $L$. mexicana mexicana, L. mexicana amazonensis y $L$. d. chagasi son extremamente intensas en $L u$. longipalpis, infectados experimentalmente con promastigotas o amastigotas. Los flagelados son numerosos y se distribuyen a lo largo del intestino medio (estómago) y anterior (esófago, cardias, faringe, probóscide), emergiendo, frecuentemente, por el extremo cortado del esófago. Esta falta de adaptación para Lu. longipalpis de algunos stocks de Leishmania aislados de nuestra área de estudios ${ }^{3}$ favorece la hipotesis de la utilización de especies locales de flebótomos, como modelos de padronización biológica por el vector $^{3}$. Lainson ${ }^{10}$ dice existir una restricción natural al hospedador invertebrado (flebótomo), existiendo una rigida asociación entre la especie de flebótomo y su especie de Leishmania; por ejemplo, la asociación $L u$. umbratilis y $L$. braziliensis guyanensis. Walton $^{18}$, usando especimenes panameños de Lu. gomezi y $L u$. trapidoi, fallaron para establecer infecciones con una cepa de L. braziliensis braziliensis (M-1287) del Brasil, probablement mejor adaptada a especimenes de Lu. wellcomei.

Bioquímicamente LTB-0016 es una $L$. mexicana amazonensis. Estos resultados fueron confirmados por Mc Mahon Pratt \& David ${ }^{12}$, con la utilización de 5 anticuerpos monoclonales que se mostraron totalmente reactivos y además, por los resultados obtenidos en nuestro laboratorio, utilizando los reactivos monoclonales en la técnica de IFAT.

Sin embargo, LTB-0055 es un miembro del complejo L. mexicana, cuando examinados los padrones isoenzimáticos de 6 enzimas, pero diferencíase de $L$. mexicana amazonensis con G6PD.

Inmunológicamente esta mezcla de caracteristicas fenotípicas exhibidas por LTB-0055 se evidenciarion mejor, cuando Mc Mahon Pratt \& David ${ }^{12}$ analizaron los resultados obtenidos con 16 anticuerpos monoclonales. Cinco de esos anticuerpos especie y subespecie especificos para $L$. mexicana amazonensis (incluso para LTB-0016 - Três Braços 04) reaccionaron positivamente. El análisis de LTB-0055 y los 11 anticuerpos monoclonales específicos para 
Cuba CAC, Barreto AC, Marsden PD. Leishmania mexicana: aspectos taxonómicos y rareza de la infección humana en Três Braços, Bahia, Brasil. Revista da Sociedade Brasileira de Medicina Tropical 17: 115-122, Jul-Set, 1984

L. braziliensis, demostró reactividad cruzada con 5 de ellos. Desde que ni la cepa padrón de $L$. mexicana amazonensis de Três Braços (LTB-0016) reaccionaron con aquellos 5 monoclonales, pensamos que éste stock de LTB-0055 es una $L$. mexicana, con nivel subespecífico indefinido hasta el presente momento, $y$ aguardando, posteriores estudios que permitan finalmente establecer su status taxonómico.

Por otro lado, nosotros no comprendemos hasta ahora el significado epidemiológico de éste hallazgo y no sabemos explicar como éste parásito actúa en la naturaleza entre sus hospedadores, vectores y reservatórios. Parece ser verdad que la infección humana es extremamente rara, a pesar de haber aislado gran número de stocks de Leishmania entre los pacientes de Três Braços.

Desconocemos que peso taxonómico tendría ésta variación isoenzimática para tipificar LTB-0055, no obstante, señalamos su presencia aguardando el aislamiento de nuevos stocks similares, en la misma área de Três Braços, que demuestren su consistente frecuencia. Al respecto, es importante frisar el concepto de la necesidad de estudiarse muchas muestras de Leishmania y muchas enzimas para tener firmeza en la caracterización bioquímica ${ }^{5}$.

\section{SUMMARY}

In a study of 51 stocks of Leishmania isolated from patients with cutaneous leishmaniasis in Três Braços, Bahia, Brazil 49 were characterized as L. braziliensis braziliensis Only two L. mexicana stocks were identified. One was subspeciated as L. mexicana amazonensis. The other differed from this species and to date remains unspeciated. The biological parameters, isoenzyme analysis and monoclonal antibody findings relating to these two strains are discussed. Transmission of parasites of the mexicana complex to man have been rare in this region during the 8 years of study of the human infection.

Key words: Leishmania mexicana L. mexicana amazonensis Human transmission. Taxonomic aspects.

\section{AGRADECIMIENTOS}

A los Drs. Ralph Lainson, J. J. Shaw y Michael Miles por las facilidades prestadas en Belem, Instituto Evandro Chagas. A Alejandro Vexenat, por la ayuda técnica con los flebótomos en Brasilia.

\section{REFERENCIAS BIBLIOGRÁFICAS}

1. Barreto AC, Peterson N, Rosa AC, Cuba CC, Vexenat JA, Marsden PD. Caracterização de estoques de Leishmania isolados de cào e de roedor (Proechimys inheringi denigratus) da região sudeste da Bahia, através de anticorpos monoclonais. XI Congresso da Sociedade Brasileira de Medicina Tropical, Salvador, Bahia, 1984.

2. Bradley DJ. Genetic control of natural resistence to Leishmania donovani infection. Nature 283: 72-73, 1974.

3. Cuba CA, Vexenat AJ, Llanos EA, Marsden PD, Barreto AC, Rosa AC. Experimental infections of wild caught specimens of Lutzomyia $(\mathrm{N})$ whitmani (Diptera. Psychodidae) and their use for Leishmania identification. IX Reunião Anual de Pesquisa Básica em Doença de Chagas, Caxambu, MG, p. 89, 1982.

4. Cuba CA. Leishmaniose tegumentar em àrea endèmica do Estado da Bahia, Brasil. Caracterização e classificaçào de Leishmania do homem e do cão domésticoe aspectos comportamentais de $L$. braziliensis braziliensis. Tese de Doutorado. Universidade Federal de Minas Gerais, Belo Horizonte, MG, 1983.

5. Godfrey DG. Biochemical characterization in the taxonomy of parasitic Protozoa. In: Biochemical characterization of Leishmania. Proceedings of a Workshop Pan American Health Organization, Washington, D.C., December 1980, pp. 63-71, 1982.

6. Lainson R, Shaw JJ. Leishmania and Leishmaniasis of the New World, with particular reference to Brazil. Bulletin of the Pan American Health Organization, 7: 1-19, 1973.

7. Lainson R, Ward RD, Shaw JJ. Leishmania in flebo tomid sandflies VI. Importance of hindgut development in distinguishing between parasites of the Leishmania mexicana and $L$. braziliensis complexes. Proceedings of the Royal Society 199: 309-320, 1977.

8. Lainson $\mathrm{R}$, Shaw JJ. The role of animals in the epidemiology of South American leishmaniasis. In: Biology of Kinetoplastida. Vol. II Lumsden WHR \& Evans, D.A. (eds). Academic Press, London, pp. 1-116, 1979.

9. Lainson R. Epidemiologia e ecologia de leishmaniose tegumentar na Amazónia. Hiléia Médica, Belém 3: $35-40,1981$.

10. Lainson R. The American Leishmaniases: some observations on their ecology and epidemiology. Transactions of the Royal Society of Tropical Medicine \& Hygiene 77: 569-596, 1983. 
Cuba CAC, Barreto AC, Marsden PD. Leishmania mexicana: aspectos taxonómicos y rareza de la infección humana en Três Braços, Bahia, Brasil. Revista da Sociedade Brasileira de Medicina Tropical 17: 115-122. Jul-Set, 1984

11. Marsden P, Cuba CC, Barreto AC, Sampaio RN, Rocha RAA. Nifurtimox in the treatment of South American Leishmaniasis. Transactions of the Royal Society of Tropical Medicine \& Hygiene 75: 556-559, 1979.

12. Mc Mahon-Pratt D, David JR. Monoclonal antibodies that distinguish between New World species of Leishmania. Nature 291: 581-583, 1981.

13. Miles MA, Povoa MM, Souza AA, Lainson R, Shaw JJ. Some methods for the enzymic characterization of Latin American Leishmania with particular reference to Leishmania mexicana amazonensis and subspecies of Leishmania hertigi. Transactions of the Royal Society of Tropical Medicine \& Hygiene 74: 243-252, 1980.

14. Mayrink W, Williams $P$, Coelho MV, Dias M, Vianna Martins A, Magalhães PA, Da Costa CA, Falcão AR, Mello MN, Falcão AL. Epidemiology of dermal leishmaniasis in the Rio Doce Valley State of Minas Gerais, Brazil. Annals of Tropical Medicine and Parasitology 73: 123-137, 1979.

15. Perez H. Factors influencing the host response to Leishmania mexicana In: Cytopathology of parasitic disease. Pitman Books, London Ciba Foundation Symposium 99, pp. 157-173, 1983.
16. Shaw JJ, Lainson R. Leishmaniasis in Brasil. XI Observations on the morphology of Leishmania of the braziliensis and mexicana complexes. Journal of Tropical Medicine and Hygiene 79: 9-13, 1976.

17. Shaw JJ. Taxonomy of the Genus Leishmania: Traditionalist's view and modern concepts. In: Biochemichal characterization of Leishmania. Proceedings of a Workshop, Pan American Health Organization, Washington, pp. 9-24, 1982.

18. Walton BC, Intermill RW, Hajduk ME. Differences in biological characteristics of three Leishmania isolates from patients with espundia. American Journal of Tropical Medicine and Hygiene 26: 850-855, 1977.

19. Walton BC, Shaw JJ, Lainson R. Observations on the "in vitro" cultivation of Leishmania braziliensis. The Joumal of Parasitology 63: 1118-1119, 1977.

20. Ward RD, Lainson R, Shaw JJ. Some methods for membrane feeding of laboratory reared neotropical sandflies (Diptera, Psychodidae). Annals of Tropical Medicine and Parasitology 72: 269-276, 1978. 